\title{
Anterior Petrosal Approach for the Resection of a Petrous Apex Meningioma with Tentorial Infiltration
}

\author{
Diego Mendez-Rosito ${ }^{1}$ \\ ${ }^{1}$ Department of Neurosurgery, Centro Médico Nacional 20 de \\ Noviembre, ISSSTE, Mexico City, Mexico \\ J Neurol Surg B 2019;80(suppl S3):S304.
}

\begin{abstract}
Address for correspondence Diego Mendez-Rosito, MD, Department of Neurosurgery, Centro Médico Nacional 20 de Noviembre, ISSSTE, Felix Cuevas, Mexico City 01900, Mexico (e-mail: diegomendezrosito@gmail.com).
\end{abstract}

Conflict of Interest None declared.
Objective The anterior petrosal approach is an extension of the middle fossa approach, characterized by drilling of the posteromedial triangle of the middle fossa. Drilling the Kawase's rhomboid creates a surgical corridor to the posterior fossa after splitting the tentorium. We present a case of a petrous apex meningioma invading the tentorium and causing trigeminal neuralgia.

Results The patient was positioned in a Mayfield with the head rotated. A frontotemporal incision was done. A basal craniotomy was done to allow epidural dissection. The anatomical landmarks were identified. The surgical video is analyzed together with cadaveric dissections to highlight landmarks when doing an anterior petrosectomy. The tentorium was identified and the infiltrated region was coagulated and removed. The tentorium was sharply sectioned until the free edge of the tentorium was opened. The tumor in the petrous apex was identified and removed. The trigeminal nerve was decompressed and a gross total resection was achieved with resolution of the symptoms.

Keywords

- anterior petrosal approach

- meningioma

- tentorium

- petrous apex

- trigeminal neuralgia
Conclusion The anterior petrosal approach is a useful corridor to remove tumors in the petrous apex that infiltrate the tentorium. A thorough knowledge of the anatomical landmarks is crucial to identify and delineate the limits of the Kawase's rhomboid. After evaluating different surgical corridors, the anterior petrosal approach allows a gross total resection including the removal of the infiltrated tentorium and a resolution of the symptoms.

The link to the video can be found at: https://youtu.be/p4KPUnM_bww.

\author{
None declared.
}

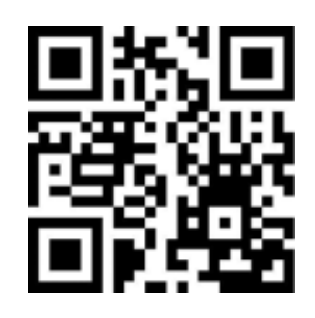

received

May 25, 2018

accepted after revision

November 11, 2018

published online

February 4, 2019 